\title{
Mobile Phone-Based Teleconsultation Service Utilization and Associated Factors Among Healthcare Professionals in Primary Hospitals of West Gojjam Zone, Northwest Ethiopia: A Cross- Sectional Study
}

Mequanint Nakachew Kasie ( $\square$ mequanint2123@gmail.com )

Department of Health Informatics, School of Public Health, College of Medicine and Health Science, Dilla University, Dilla

\section{Habtamu Alganeh Guadie}

School of Public health, College of Medicine and Health Science, BahirDar University, BahirDar,

Kassahun Dessie Gashu

Department of Health Informatics, Institute of Public Health, College of Medicine and Health Science, University of Gondar, Gondar,

\section{Research Article}

Keywords: Teleconsultation, Mobile phone, associated factors, Healthcare professionals, Northwest Ethiopia

Posted Date: June 17th, 2021

DOl: https://doi.org/10.21203/rs.3.rs-601821/v1

License: (c) (1) This work is licensed under a Creative Commons Attribution 4.0 International License. Read Full License 


\section{Mobile phone-based teleconsultation service utilization and associated factors among healthcare professionals in primary hospitals of West Gojjam Zone, Northwest Ethiopia: a cross- sectional study.}

Mequanint Nakachew Kasie ${ }^{1 *}$, Habtamu Alganeh Guadie ${ }^{2}$, Kassahun Dessie Gashu ${ }^{3}$

${ }^{1}$ Department of Health Informatics, School of Public Health, College of Medicine and Health Science, Dilla University, Dilla, Ethiopia

${ }^{2}$ School of Public health, College of Medicine and Health Science, BahirDar University, BahirDar, Ethiopia

${ }^{3}$ Department of Health Informatics, Institute of Public Health, College of Medicine and Health Science, University of Gondar, Gondar, Ethiopia

${ }^{*}$ Correspondence: Mequanint Nakachew Kasie

Gmail: mequanint2123@gmail.com / mequanint333@gmail.com

Tel: +2519 21156847

Dilla, Ethiopia 


\section{Abstract}

Background-Teleconsultation with a mobile phone is one form of telemedicine which can be easily practiced since most healthcare professionals own mobile phone. Many patients are forced to wait for a while before they get to see a specialist. Patients also forced unnecessary transport and lost their time and money during the referral process. Therefore this study aims to determine the level of mobile phone-based teleconsultation service utilization and identify associated factors among healthcare professionals in primary hospitals.

Methods: Institutional-based cross-sectional study design was employed. A structured, pre-tested questionnaire was used for data collection. Binary logistic regression has been used to assess factors associated with the outcome variable.

Result: A total of 388 healthcare professionals ( $92 \%$ response rate) were involved in the study from 8 primary hospitals. Mobile phone-based teleconsultation service utilization was found 106(27.3\%). Healthcare professionals who had good knowledge of teleconsultation were 6.6 times ( $\mathrm{AOR}=6.6,95 \% \mathrm{Cl}: 1.46-29.93$ ) and those who perceive teleconsultation as useful were 7.2 times ( $A O R=7.2,95 \% \mathrm{Cl}$ : $2.036-25.589$ ) more likely to utilize mobile phone-based teleconsultation service than their counterparts.

Conclusion: The finding has shown that healthcare professionals' level of mobile phone-based teleconsultation service utilization was low. Internet connectivity, participants' knowledge towards teleconsultation, perceived usefulness, and peer influence were found to be significantly associated with mobile phone-based teleconsultation service utilization in primary hospitals.

Key Words: Teleconsultation, Mobile phone, associated factors, Healthcare professionals, Northwest Ethiopia 


\section{Background}

Teleconsultation is electronic communication between physicians and clients, or among healthcare professionals for diagnostic, therapeutic advice, or educational purposes over distance (1). Teleconsultation with a mobile phone is one form of telemedicine which can be easily practiced since most healthcare professional owns a mobile phone. To improve access and quality of healthcare teleconsultation has a vital role in providing healthcare delivery service when distance is a critical factor (1).

Teleconsultation bridges the knowledge gaps between healthcare providers resulting in standardized treatment across organizational boundaries. It facilitates access to specialist health services and support. It allows patients to stay in their homes, schools, or community centers and avoids or reduces the number of travels to specialized hospitals $(2,3)$. This means more time spent with supporters (family and friends), less stress from travel to unknown hospitals and locations, and less time away from work.

The goal of teleconsultation is to eliminate the barriers of distance and promote equitable access to health services to remote areas where face-to-face consultations were unavailable (2).

This study was conducted in Primary hospitals (one of the primary healthcare units $(\mathrm{PHCU}))$, the lowest level in Ethiopia three tires health care systems. Healthcare delivery processes in primary hospitals were practiced by healthcare professionals with a low level of education and high-level medical diagnosis instruments were not available as tertiary hospitals so that they need to consult with specialists and sinners working abroad to solve patients' health problems accurately.

In many developing countries, including Ethiopia, patients usually face untrained medical specialists, especially in rural areas. Many patients are forced to wait for a while before they get to see a specialist. In many cases, healthcare providers are forced to refer patients with complicated medical cases from primary healthcare centers to tertiary centers due to a lack of available specialists $(4,5)$. In Ethiopia, it is unlikely to offer specialized care in rural communities with having few specialists. With teleconsultation, a specialist can provide care to a patient without either of them having to leave home. 
There are many barriers like geographical access and affordability of healthcare services, especially for rural communities. Patients are forced for unnecessary transport, fear of travel to unknown hospitals, and lost time. The cost of healthcare has always become a crucial issue. Catastrophic health expenditure is posing a threat to a household's financial ability (6).

Therefore, in this study, the level of teleconsultation service utilization and associated factors were assessed among healthcare professionals in primary hospitals.

\section{Methods and materials}

\section{Study Design}

An Institutional based cross-sectional study was conducted to assess healthcare professionals' level of mobile-based teleconsultation service utilization.

\section{Study setting and period}

The study was conducted in West Gojjam zone primary hospitals from March, 05 to April 25 , 2020. This study focuses on primary hospitals. Because most healthcare professionals in primary hospitals have a low level of education. So, if they use teleconsultation services with specialists and other experienced care providers, they will provide better healthcare to the clients and patients. West Gojjam zone is one of the zones in the Amhara region and its capital is Finote-Selam which is $385 \mathrm{~km}$ from Addis Abeba capital city of Ethiopia and 176km from Bahir Dar capital of the Amhara region.

\section{Source and Study population}

All healthcare professionals in primary hospitals of the west Gojjam zone were taken as the source population.

Selected healthcare professionals in primary hospitals of the West Gojjam zone were taken as the study population.

\section{Inclusion and exclusion criteria}

Healthcare professionals working in primary hospitals of the West Gojjam zone were included in the study. 
Healthcare professionals of primary hospitals in the West Gojjam zone but those who were on annual leave, leave for long-term education, leave due to illness, and female healthcare providers who are on maternity leave were excluded from the study.

\section{Variables of study}

\section{Dependent variable}

Teleconsultation service utilization

\section{Independent variables}

Behavioral factors: knowledge towards teleconsultation and Attitude towards teleconsultation.

Sociodemographic factors: age, sex, experience, profession, education level

Technological factors: Perceived ease of use, perceived usefulness, type of mobile, Internet connection, Digital literacy

Social factor: peer influence, Patient expectation

Organizational Factors: Internet availability, Training

\section{Operational definition}

Perceived usefulness - Perceived usefulness is defined as the degree to which healthcare professionals believe that using a teleconsultation would enhance his or her job performance. Twelve item questions with a five-point Likert scale from not useful at all to very useful were used to measure the perceived usefulness of study participants towards teleconsultation (15).

Perceived ease of use- refers to the degree to which healthcare professionals believe that using a teleconsultation would be free of effort and/or freedom from difficulty or great effort. Twelve item questions with a five-point Likert scale from very easy to very difficult were used to measure the perceived ease of use of study participants towards teleconsultation (15).

Mobile phone-based teleconsultation service utilization- is the use of mobile phonebased teleconsultation services at least once a week (10). 


\section{Sample size determination}

The sample size is calculated using single population proportion formula:

$$
\mathrm{n}=\left(\mathrm{Z}^{\alpha / 2}\right)^{2} \times \mathrm{pq} \quad, \quad \mathrm{n}=(1.96)^{2} \times 0.5 \times 0.5=384
$$

$$
\mathrm{d}^{2} \quad 0.05^{2}
$$

With the following assumptions:

$\mathrm{n}=$ the required sample size

$Z=$ the value of standard normal distribution corresponding to $\alpha / 2=1.96$

$p=50 \%$

$q=1-p$,

$d=$ is the margin of error $5 \%(0.05)$,

With $10 \%$ none response rate, $10 \%$ of $384=38$, Final sample size $\mathbf{n}=\mathbf{4 2 2}$

\section{Sampling procedure}

Stratified sampling was used by considering clinical departments as strata. Participants were selected proportionally from each hospital as well as from each profession.

\section{Data collection tools}

A structured questionnaire adapted from different articles was used to collect data about sociodemographic characteristics of respondents, utilization of teleconsultation, and associated factors (7-11). The questionnaire had a close-ended nature. It also includes the Likert scale, yes/no, and lists of response options. The questionnaire was first prepared in English and translated into the local language Amharic. It was pre-tested for reliability before actual data collection.

\section{Data collection procedure}

Data was collected by 8 trained data collectors guided by two supervisors from March, 05 to April 25, 2020. Data collectors were health information technology (HIT) professionals. The training was given to data collectors.

\section{Data quality control}

To control the quality of data, training was given to data collectors before the actual data collection. Properly designed and pre-tested questionnaires have been used. After data 
collection, questionnaires were reviewed and checked for completeness and the data was cleaned to check for errors and missed values, and any errors identified have been corrected.

\section{Data management and analysis}

The data entry form was designed using epi info 7.2 version and analysis was conducted using SPSS version 20. Hosmer-Lemeshow tests were used to check model fit. Then binary logistic regression has been used to assess factors associated with teleconsultation service utilization. The normality assumption of data distribution was also checked.

The results were presented in the form of tables, figures, and text using frequencies and summary statistics to describe the study population with relevant variables. A P-value less than or equal to 0.05 has been taken as a cut of value to be significant. The odds ratio was used to measure the strength of association between predictor variables and the outcome variable.

\section{Results}

\section{Characteristics of study participants}

A total of 388 healthcare professionals from 8 primary hospitals were complete all the questionnaires (92\% response rate) from 422 samples. From the total $(n=388)$ respondents $239(61.6 \%)$ were male (Table 1). Most study participants Levels of education were BSC degree (57.5\%) followed by Diploma (23.2\%) (Table 1).

Table 1. Characteristics of study participants

\begin{tabular}{llrr}
\hline \multicolumn{2}{c}{ Variables } & Frequency & Percent (\%) \\
\hline Sex & male & 239 & 61.6 \\
& female & 149 & 38.4 \\
Age & $<30$ & 284 & 73.2 \\
& $30-40$ & 88 & 22.7 \\
\multirow{3}{*}{ Type of } & $>40$ & 16 & 4.1 \\
profession & Physician & 59 & 15.2 \\
& Nursing & 177 & 45.6 \\
& Midwifery & 53 & 13.7 \\
& Laboratory & 50 & 12.9 \\
& Pharmacist & 49 & 12.6 \\
\hline
\end{tabular}




\begin{tabular}{llrr}
\hline $\begin{array}{l}\text { Level of } \\
\text { education }\end{array}$ & Specialty & 1 & .3 \\
& General & 59 & 15.2 \\
& practitioner(GP) & & \\
& MSc Degree & 15 & 3.9 \\
& BSc Degree & 223 & 57.5 \\
& Diploma & 90 & 23.2 \\
Experience & 1-5 year & 241 & 62.1 \\
& 5-10 year & 113 & 29.1 \\
& $>10$ year & 34 & 8.8 \\
\hline
\end{tabular}

There're barriers to healthcare professionals not use teleconsultation in their day-to-day healthcare delivery process. Some of the reasons were ICT infrastructure, poor internet connectivity, low awareness and knowledge, financial problems, and lack of expert support (figure 1).

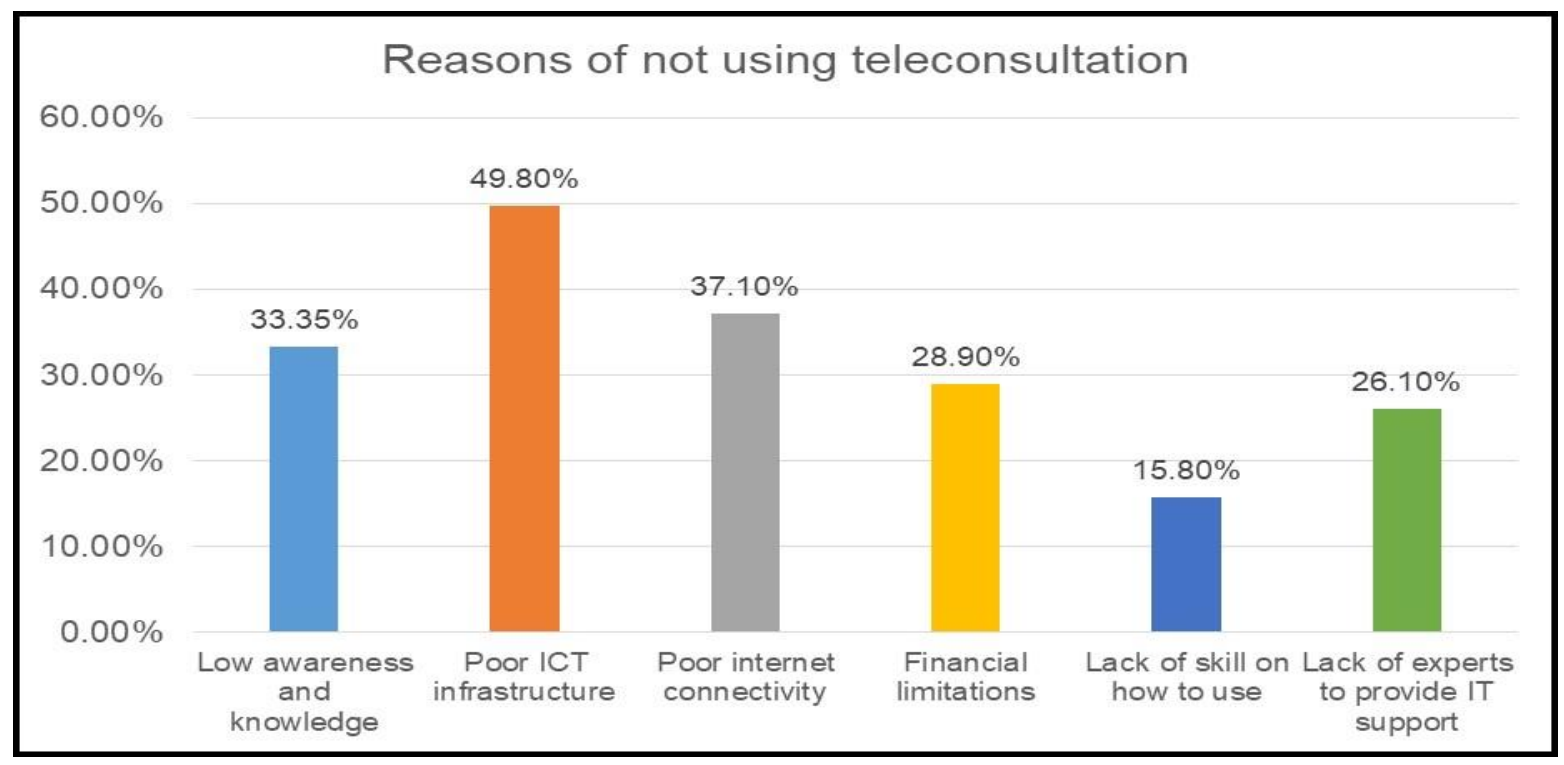

Figure 1: Reasons why healthcare professionals in primary hospitals of west Gojjam zone did not use teleconsultation, 2020. 


\section{Mobile phone-based teleconsultation service utilization}

Healthcare professionals' mobile phone-based teleconsultation service utilization was found 106 (27.3\%). Most healthcare professionals conduct teleconsultation with peer friends $(74.7 \%)$ followed by specialists working in the same hospital $(36.8 \%)$ although they had consulted with different bodies (Table 2). The purpose of healthcare professionals using teleconsultation was for accurate diagnosis and treatment (50.5), drug Information (45.3), and surgical procedures (29.3). One healthcare professional had consulted for more than one different purpose (Table 2).

Table 2: The purpose of teleconsultation and with whom healthcare professionals in primary hospitals were consulted, 2020.

\begin{tabular}{ll}
\hline Variables & No (\%) \\
\hline $\begin{array}{l}\text { from whom healthcare } \\
\text { professionals consult with }\end{array}$ & \\
Specialists from other hospitals & $39(20.5)$ \\
Specialists from the same hospital & $70(36.8)$ \\
Peer & $142(74.7)$ \\
\hline Purpose & $48(25.3)$ \\
Referral & $50(26.3)$ \\
Laboratory & $24(12.6)$ \\
Radiological & $86(45.3)$ \\
Drug Information & $96(50.5)$ \\
For accurate diagnosis & $56(29.5)$ \\
For surgical procedures & \\
\hline
\end{tabular}

Healthcare professionals have shared different types of data during the teleconsultation process. Most of them have shared audio data/audio call 134 (70.5\%) followed by text message $86(45.3 \%)$ although one healthcare professionals had shared different types of data during teleconsultation. 
Teleconsultation service utilization was higher in midwifery professionals at $39.6 \%$ and low in laboratory professionals. It is also higher in low-level education professionals (BSC and diploma, 29\% each) than higher-level education professionals (table 3 ).

Table 3: Level of teleconsultation service utilization among healthcare professionals in primary hospitals west Gojjam zone concerning different characteristics of study participants, $2020(n=388)$.

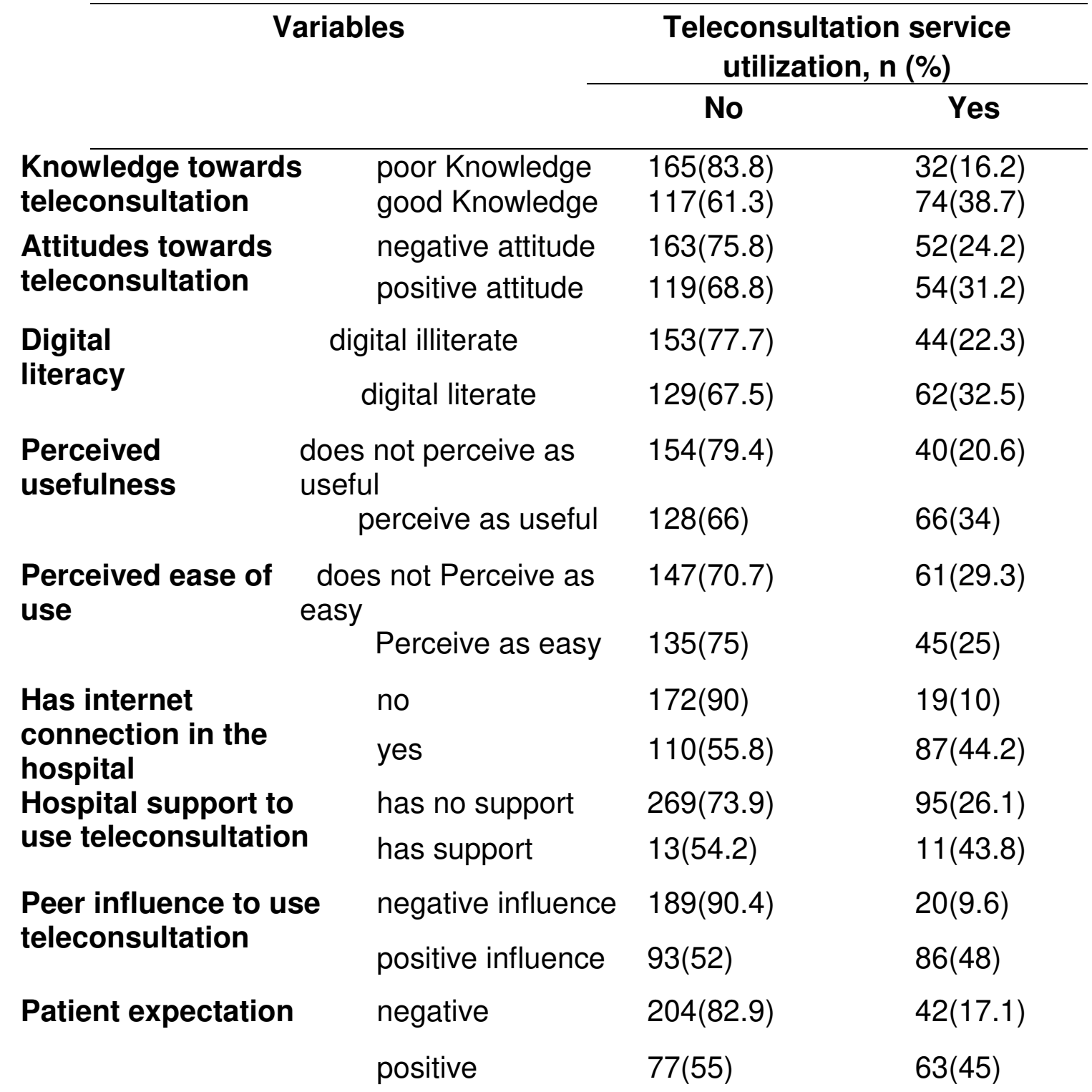




\section{Factors associated with mobile phone-based teleconsultation service utilization}

Bi-variable logistic regression analysis of possible explanatory variables with mobile phone-based teleconsultation service utilization was carried out. Variables in the bivariable analysis with $p$-value $<0.020$ were further taken into multi-variable analysis using the enter method. The multi-variable logistic regression analysis revealed that the following factors were significantly associated with mobile phone-based teleconsultation service utilization: knowledge ( $p=0.017)$, perceived usefulness $(p=0.002)$; peer influence $(p<0.001)$, and internet access in hospital $(p<.001)$ (Table 4).

Healthcare professionals who had good knowledge of teleconsultation were 6.6 times (AOR=6.6, 95\% Cl: 1.46-29.93) more likely to utilize teleconsultation services than their counterparts.

Healthcare professionals who perceive teleconsultation as useful for healthcare delivery were 7.2 times $(\mathrm{AOR}=7.2,95 \% \mathrm{Cl}: 2.036-25.589)$ more likely to utilize teleconsultation service than their counterparts.

Healthcare professionals who work in hospitals with internet access were 3.54 times (AOR=3.54, 95\% Cl: $1.885-6.657$ ) more likely to utilize teleconsultation service than their counterparts.

Healthcare professionals who had co-workers with positive influence towards teleconsultation utilization were 3.5 times ( $A O R=3.5,95 \% \mathrm{Cl}: 1.834-6.619)$ more likely to utilize teleconsultation service than their counterparts.

Table 4: logistic regression analysis of factors with teleconsultation utilization among healthcare professionals in primary hospitals of West Gojjam Zone, North West Ethiopia $(n=388)$.

\begin{tabular}{ccccccc}
\hline \multirow{2}{*}{ Factors } & \multicolumn{2}{c}{$\begin{array}{c}\text { Teleconsultation } \\
\text { service utilization }\end{array}$} & \multicolumn{2}{c}{ OR 95\% C.I } & \\
\cline { 2 - 3 } & Yes n (\%) & No $\mathbf{n}(\%)$ & COR & AOR & P-value \\
\hline $\begin{array}{c}\text { Digital literacy } \\
\text { Digital literate }\end{array}$ & $58(32.7)$ & $119(67.3)$ & $1.66(1.06-2.59)$ & $1.72(.89-3.32)$ & .106 \\
\hline
\end{tabular}




\begin{tabular}{|c|c|c|c|c|c|}
\hline \multicolumn{6}{|l|}{$\begin{array}{c}\text { Digital illiterate } \\
\text { Perceived usefulness }\end{array}$} \\
\hline Yes & $103(31.8)$ & $221(68.2)$ & $9.48(2.91-30.92)$ & $7.20(2.04-25.59)$ & $0.002^{*}$ \\
\hline No & $3(4.7)$ & $61(95.3)$ & 1 & 1 & \\
\hline \multicolumn{6}{|l|}{ Perceived ease of use } \\
\hline Yes & $99(29.6)$ & $236(70.4)$ & $2.76(1.20-6.312)$ & $1.14(.39-3.37)$ & .814 \\
\hline No & $7(13.2)$ & $46(86.8)$ & 1 & 1 & \\
\hline \multicolumn{6}{|l|}{ Knowledge } \\
\hline Good & 104(35.4) & $190(64.6)$ & $4.42(2.13-9.17)$ & $6.6(1.46-29.93)$ & $0.017^{*}$ \\
\hline \multicolumn{5}{|l|}{ Peer influence } & \\
\hline Yes & $86(48)$ & $93(52)$ & $8.74(5.06-15.09)$ & $3.50(1.83-6.62)$ & $<0.001^{*}$ \\
\hline No & $20(9.6)$ & $189(90.4)$ & 1 & 1 & \\
\hline \multicolumn{6}{|l|}{ Patient expectation } \\
\hline positive & $63(45)$ & $77(55)$ & $3.97(2.48-6.36)$ & 1.83(.99-3.35) & .051 \\
\hline Negative & $42(17.1)$ & 04(82.9) & 1 & 1 & \\
\hline \multicolumn{6}{|l|}{ Internet connection } \\
\hline Yes & $87(44.1)$ & $110(55.2)$ & 7.16(4.13-12.42) & $3.54(1.89-6.66)$ & $<0.001^{*}$ \\
\hline No & $19(10)$ & $172(90)$ & 1 & 1 & \\
\hline \multicolumn{6}{|l|}{ Hospital support } \\
\hline Yes & $11(45.8)$ & $13(54.2)$ & $2.39(1.04-5.53)$ & $1.021(.34-3.05)$ & 971 \\
\hline No & $95(26.1)$ & $269(73.9)$ & 1 & 1 & \\
\hline
\end{tabular}

${ }^{*} P<0.05$ (statistically significant), COR-Crude Odds Ratio, AOR-Adjusted Odds Ratio

\section{Discussion}

The study has shown that $106(27.3 \%)$ of healthcare professionals have utilized mobile phone-based teleconsultation services for the healthcare delivery process. This figure is lower than the finding in Brazil, the use (51\%) of teleconsultation system in primary care, 2015 (9), and in Italy, teleconsultation service use among general practitioners (52\%) to improve healthcare in rural areas (1). This may be due to the study participants were only Doctors while the current study involved all low, middle, and higher-level healthcare professionals. It may also be due to poor ICT infrastructure and internet connectivity in hospitals in Ethiopia.

Knowledge about teleconsultation, perceived usefulness, peer influence, and internet connection in the hospital were among significantly associated variables with mobile phone-based teleconsultation service utilization. 
This study has shown that 294 (75.8\%) of healthcare professionals had good knowledge towards teleconsultation, which has shown that having good knowledge (AOR=6.6(95\% C.I. (1.46-29.93) $p=0.017$ ) towards teleconsultation was facilitating factor to utilize teleconsultation service in healthcare delivery process. This is supported by a qualitative study conducted in Canada, 2017(12). This finding is higher than residents' knowledge of teleconsultation in Isfahan AL-Zahra hospital, Iran, 2020 (13). This variety may be due to different participants and settings, the current study includes different professions not only residents.

The current study has shown that healthcare professionals who perceived teleconsultation as useful were $83.5 \%$. Perceived usefulness was (AOR=7.2(95\% C.I. (2.036-25.589), $P=0.002$ ) found as a facilitating factor for teleconsultation utilization. This finding is supported by a mixed Study in Malaysia (6) and a qualitative study conducted in South Africa, 2018, that is medical specialists had a positive perception for the usefulness teleconsultation had used for educational and diagnosis-related advice (14). It is also supported by healthcare providers' technology acceptance theory (15. This is maybe due to that if healthcare professionals perceive teleconsultation as useful, they have intended to use teleconsultation in their day-to-day healthcare delivery process.

Although ICT infrastructure and internet connectivity were poor (82.8\%) in primary hospitals of the West Gojjam zone, result shown that healthcare professionals from hospitals with internet connectivity were $197(50.8 \%)$. Internet connection was found as a facilitating factor to utilize teleconsultation for the healthcare delivery process ( $A O R=3.54$, (95\% Cl: $1.885-6.657, p<0.001)$. This may be due to if there were internet connection in hospitals, Healthcare professionals have got the opportunity to use mobile apps and able to synchronously connect easily with peers and specialists from remote or tertiary hospitals.

Healthcare professionals who have positive co-workers/ social support towards teleconsultation were 179 (46.1\%). Positive peer influence were (46.1\%), AOR=3.5, (95\% Cl: 1.834-6.619, $\mathrm{p}<0.001)$ facilitating factor for teleconsultation service utilization. This finding is supported by healthcare providers technology acceptance theory (15) and comparative study of healthcare provider's acceptance and use of technology in the 
United States and China, 2014, which is social influence affects intention to use telehealth technologies (8)

\section{Conclusion}

The finding has shown that the level of mobile phone-based teleconsultation service utilization was low. Internet connectivity, participants' knowledge towards teleconsultation, perceived usefulness, and peer influence were found to be significantly associated with teleconsultation service utilization in primary hospitals.

\section{Declarations}

\section{Ethical consideration}

Ethical clearance was obtained from the Institutional Review Board (IRB) of the University of Gondar, College of Medicine and Health Science, and institute of public health with Ref № IPH/837106/2012. Informed consent was obtained from each study participant. The study was conducted following the Declaration of Helsinki. Data collected were anonymized so that participants' privacy was protected. All were kept confidential and used for study purposes only.

\section{Consent for publication}

Not applicable

\section{Availability of data}

The datasets used and/or analyzed during the current study available from the corresponding author on reasonable request.

\section{Competing Interests}

Authors have no competing interest in this work.

\section{Funding}

The is no funding to report. 


\section{Author's contribution}

All authors contributed to the research design, data preparation, analysis, discussion, and manuscript writing.

\section{Acknowledgment}

We would like to express our deepest gratitude to the data collectors and study participants for their positive support for the achievement of the research paper.

\section{References}

1. Zanaboni et al. Teleconsultation service to improve healthcare in rural areas. Biomedical Center, 2009, 9(1):238.

2. Dorsey et al. State of Telehealth. New England Journal of Medicine, 2016, 375(2):154161.

3. Nilsen \& Moen, Teleconsultation-collaborative work and opportunities for learning across organizational boundaries. Journal of Telemedicine and Telecare, 2008, 14(7):377-380.

4. Abushaar $L$ and Ismail $A$, Acceptance of Teleconsultation Among Doctors and The Determinant Factors in Teaching Hospital in Malaysia. Atlantis Press. 2017, 9(1)

5. Maarop $\mathrm{N}$ and Win KT, Understanding the Need of healthcare providers for teleconsultation and technological attributes in relation to the acceptance of teleconsultation in Malaysia: A mixed-methods study. Journal of medical systems. 2012,36(5): 2881-2892

6. Kifle YA and Tilahun N, Cost-effectiveness analysis of clinical specialist outreach as compared to referral system in Ethiopia. Biomedical Center, 2010;8(1):13

7. Gagnon MP et al. Using a modified technology acceptance model to evaluate healthcare professionals' adoption of a new telemonitoring system. Telemedicine and e-Health, 2012, 18(1):54-59.

8. Venkatesh $V$ and Zhang $X$, Unified Theory of Acceptance and Use of Technology. Journal of Global Information Technology Management, 2014, 13(1):5-27. 
9. Alkmim MB et al. Factors associated with the use of a teleconsultation system in Brazilian primary care. Telemedicine and e-Health. 2015;21(6):473-483.

10. Tamiru.B, Utilization of telemedicine in tikur anbessa specialized hospital, Addis Ababa, Ethiopia Addis Ababa University, June 2013.

11. Langbecker et al. Using survey methods in telehealth research. Journal of Telemedicine and Telecare, 2017, Vol. 23 (9):770-779

12. Bello AK et al. Patient and provider perspectives on the design and implementation of an electronic consultation system for kidney care delivery in Canada: a focus group study. Biomedical Journal. 2017

13. Tavakoli et al. Assistants' awareness of teleconsultation in Isfahan AL-Zahra hospital. International Journal of Health-System and Disaster Management, Apr-Jun 2013,1(2):105.

14. Blom $L$ et al. Expectations of medical specialists about image-based teleconsultation - A qualitative study on acute burns in South Africa. PLoS One. 2018, 13(3):e0194278.

15. Fred D. D, Perceived usefulness, perceived ease of use, and user acceptance of information technology. Management Information Systems Research Center, 1989,13(3):319-140. 